\title{
DUALITY FOR BOOLEAN EQUALITIES
}

\author{
LEON LEBLANC
}

Introduction. Boolean equalities occur naturally in Logic and especially in the theory of polyadic algebras. For instance, they have been used in [2] under the name of Champ Logique. The purpose of this paper is to develop a duality theory for Boolean equalities and by doing so establish a representation theory for them.

0 . Notation. The basic notation for Boolean algebras is the standard one (for instance, see [1]). Throughout this paper, the letter $O$ will denote the two-element Boolean algebra; whenever a topology is assumed to be defined on $O$, then this topology is the discrete one. If $B$ is a Boolean algebra, then a valuation of $B$ is a homomorphism from $B$ onto $O$. We make precise the terminology we shall be using in connection with the Stone duality theory for Boolean algebras. A Boolean space is a compact Hausdorff space whose topology is generated by the clopen sets. If $X$ is a Boolean space, then the $d u a l$ algebra of $X$ is the Boolean algebra of clopen sets of $X$. If $B$ is a Boolean algebra, then the dual space of $B$ is the Boolean space $X$ of all valuations of $B$ (the topology on $X$ is the topology generated by all sets of the form $\{v: v p=1\}$ where $p$ belongs to $B$ ).

1. Definition and generalities. A Boolean equality on a set $\Delta$ is a pair $(B, E)$ where $B$ is a Boolean algebra and $E$ is a mapping from $\Delta \times \Delta$ into $B$ so that, for all $s, t, u$ in $\Delta$,

$$
\begin{aligned}
& E(s, s)=1, \\
& E(s, t)=E(t, s), \\
& E(s, u) \wedge E(u, t) \leqq E(s, t),
\end{aligned}
$$

and

$$
B \text { is generated by the range of } E \text {, i.e., by } E(\Delta \times \Delta) \text {. }
$$

In the sequel, it will be convenient to view an equivalence relation on $\Delta$ as a mapping $\phi$ from $\Delta \times \Delta$ into $O$ so that $\phi$ satisfies (1.1), (1.2) and (1.3). Clearly, if $\phi$ is an equivalence relation on $\Delta$, then $(O, \phi)$ is a Boolean equality on $\Delta$. Suppose $(B, E)$ and $(\tilde{B}, E)$ are Boolean equalities on a set $\Delta$. A homomorphism $f$ from $B$ into $\tilde{B}$ shall be called an equality homomorphism if $f E=\tilde{E}$, i.e., if $f E(s, t)=\tilde{E}(s, t)$ for all $s$ and $t$ in $\Delta$. It is easy to see that an equality homomorphism from $B$ into $\widetilde{B}$ is necessarily onto. The Boolean equalities $(B, E)$ and $(\widetilde{B}, \tilde{E})$

Received by the editors December 19, 1960. 
are said to be isomorphic if there exists an equality isomorphism from $B$ onto $\widetilde{B}$. If $M$ is an ideal in $B, \widetilde{B}=B / M, f$ is the natural projection from $B$ onto $\widetilde{B}$ and $\tilde{E}(s, t)=f E(s, t)$ for all $s, t$ in $\Delta$, then $(\widetilde{B}, \tilde{E})$ is a Boolean equality on $\Delta ;(\widetilde{B}, \widetilde{E})$ shall be called a quotient of $(\mathrm{B}, E)$.

2. Duality. Let $\Delta$ be a nonempty set. In this section, we shall establish a one-to-one correspondence between Boolean equalities on $\Delta$ and certain sets of equivalence relations on $\Delta$. As we shall see, this correspondence obeys all the laws of a genuine duality theory. We begin by introducing a topology on the set $\Phi$ of all equivalence relations on $\Delta$. Note first that the set of all functions from $\Delta \times \Delta$ into $O$ with the product topology is a Boolean space. The topology induced on $\Phi$ shall also be referred to as the product topology. This topology is generated by the sets of the form $\{\phi: \phi \in \Phi, \phi(s, t)=1\}$ together with their complements. We have the following basic fact.

(2.1) Lemma. The set $\Phi$ of all equivalence relations on $\Delta$ together with the product topology is a Boolean space.

Proof. It suffices to show that $\Phi$ is closed in the Boolean space of all functions from $\Delta \times \Delta$ into $O$. Let $\Phi_{1}, \Phi_{2}$ and $\Phi_{3}$ be the sets of functions from $\Delta X \Delta$ into $O$ satisfying (1.1), (1.2) and (1.3) respectively. Clearly $\Phi=\Phi_{1} \cap \Phi_{2} \cap \Phi_{3}$. It suffices to show that $\Phi_{1}, \Phi_{2}$ and $\Phi_{3}$ are closed. For instance, $\Phi_{3}$ is the intersection of all sets of the form $\{\phi: \phi(s, u)=0\} \cup\{\phi: \phi(u, t)=0\} \cup\{\phi: \phi(s, t)=1\}$ as $s, u$ and $t$ run over $\Delta$; since these sets are closed, it follows that $\Phi_{3}$ is closed. The proof of the fact that $\Phi_{1}$ and $\Phi_{2}$ are closed is similar.

We are now in a position to make more precise the comment made at the beginning of this section. We shall establish a one-to-one correspondence between nonempty compact subspaces of $\Phi$ and Boolean equalities on $\Delta$. First, we define the dual Boolean equality of a nonempty compact subspace $\Psi$ of $\Phi$. The dual Boolean equality of $\Psi$ is the pair $(B, E)$ where $B$ is the dual Boolean algebra of $\Psi$ and $E$ is defined by

$$
E(s, t)=\{\phi: \phi \in \Psi, \phi(s, t)=1\}
$$

for all $s$

and $t$ in $\Delta$. It is easy to check that $(B, E)$ is indeed a Boolean equality on $\Delta$. We now define the dual equivalence relation space of a Boolean equality $(B, E)$. The dual equivalence relation space of $(B, E)$ is a subspace $\Psi$ of $\Phi$ so that $\phi \in \Psi$ if and only if there exists a valuation $v$ of $B$ for which

$$
\phi(s, t)=v E(s, t)
$$


for all $s$ and $t$ in $\Delta$. Note that if $v$ is a valuation of $B$ and $\phi$ is defined by (2.3), then $\phi \in \Phi$ and therefore $\phi \in \Psi$. We turn now to the proof of the fact that $\Psi$ is a Boolean space.

(2.4) TheOREM. If $\Psi$ is the dual equivalence relation space of a Boolean equality $(B, E)$, then $\Psi$ is homeomorphic with the dual space of $B$; in particular, this implies that $\Psi$ is a Boolean space.

Proof. Let $X$ be the dual space of $B$ and define a one-to-one mapping $\chi$ from $X$ onto $\Psi$ by

$$
\chi(v)(s, t)=v E(s, t)
$$

for all valuations $v$ in $X$ and all $s, t$ in $\Delta$. Now observe that the topology on $X$ is generated by the sets of the form $\{v: v E(s, t)=1\}$ together with their complements as $s$ and $t$ run over $\Delta$. The proof of the fact that $\chi$ is a homeomorphism is then completed after observing that the following identity holds:

$$
\chi\{v: v E(s, t)=1\}=\{\phi: \phi \in \Psi, \phi(s, t)=1\}
$$

for all $s, t$ in $\Delta$. Since $X$ is a Boolean space, it follows that $\Psi$ is a Boolean space.

We now show that the correspondence we have just established obeys the laws of duality.

(2.6) Theorem. If $(B, E)$ is a Boolean equality on $\Delta$ and $\Psi$ is the dual equivalence relation space of $(B, E)$, then the dual Boolean equality of $\Psi$ is isomorphic with $(B, E)$; if $\Psi$ is a Boolean subspace of $\Phi$ and $(B, E)$ is the dual Boolean equality of $\Psi$, then the dual equivalence relation space of $(B, E)$ is $\Psi$.

Proof. To prove the first part of the theorem, let $(\tilde{B}, \tilde{E})$ be the dual Boolean equality of $\Psi$ and let $X$ be the dual space of $B$. Let $\chi$ be the natural mapping from $X$ onto $\Psi$ defined by (2.5). If $C$ is the dual algebra of $X, f$ the natural isomorphism from $B$ onto $C, \chi_{0}$ the isomorphism from $C$ onto $\tilde{B}$ induced by $\chi$, then $\chi_{0} f$ is an isomorphism from $B$ onto $\widetilde{B}$ so that $\left(\chi_{0} f\right) E(s, t)=\tilde{E}(s, t)$ for all $s$ and $t$ in $\Delta$. It follows that $(B, E)$ and $(\widetilde{B}, \tilde{E})$ are isomorphic. To prove the second part of the theorem, assume $\Psi$ is a Boolean subspace of $\Phi$ and $(B, E)$ is the dual Boolean equality of $\Psi$. Let $X$ be the dual space of $B$ and let $g$ be the natural homeomorphism from $\Psi$ onto $X$, i.e., if $\phi \in \Psi$, then $g(\phi)$ is the unique valuation of $B$ so that, for all $p$ in $B, g(\phi) p=1$ if and only if $\phi$ belongs to the clopen set $p$. We have, for every $\phi$ in $\Psi$ and every $s, t$ in $\Delta, g(\phi) E(s, t)=\phi(s, t)$ which shows, by (2.3), that $\Psi$ is the dual equivalence relation space of $(B, E)$. 
(2.7) Corollary. Two Boolean equalities on a set $\Delta$ are isomorphic if and only if they have the same dual equivalence relation space.

Our next and final task in this section is to find necessary and sufficient conditions (in term of dual spaces) for a Boolean equality to be a quotient of another one.

(2.8) Theorem. Suppose $(B, E)$ and $(\tilde{B}, \tilde{E})$ are Boolean equalities on $\Delta$ and suppose $\Psi$ and $\tilde{\Psi}$ are their respective dual equivalence relation spaces. Then $(\tilde{B}, \tilde{E})$ is a quotient of $(B, E)$ if and only if $\tilde{\Psi}$ is a subset of $\Psi$.

Proof. Suppose there exists an equality homomorphism $f$ from $B$ onto $\tilde{B}$. Let $\phi$ in $\tilde{\Psi}$ and let $\tilde{v}$ be the unique valuation of $\widetilde{B}$ so that $\phi(s, t)=\tilde{v} \tilde{E}(s, t)$ for all $s, t$ in $\Delta$. Let $v=\tilde{v} f$. Then $v$ is a valuation of $B$ and $v E(s, t)=\phi(s, t)$ which shows that $\phi \in \Psi$. Hence $\tilde{\Psi}$ is a subset of $\Psi$. Conversely, assume $\tilde{\Psi}$ is a subset of $\Psi$. In view of the preceding theorem we may and do assume that $(\widetilde{B}, \tilde{E})$ and $(B, E)$ are the dual Boolean equalities of $\tilde{\Psi}$ and $\Psi$ respectively. Let $g$ be the inclusion mapping of $\tilde{\Psi}$ into $\Psi$; since $g$ is continuous, it induces a Boolean homomorphism $\tilde{g}$ from $B$ onto $\tilde{B}$. But

$$
g^{-1}\{\phi: \phi \in \Psi, \phi(s, t)=1\}=\{\phi: \phi \in \tilde{\Psi}, \phi(s, t)=1\}
$$

for all $s$ and $t$ in $\Delta$ which is the same as saying that $\tilde{g} E(s, t)=\tilde{E}(s, t)$ for all $s, t$ in $\Delta$. Hence $\tilde{g}$ is an equality homomorphism from $B$ onto $\tilde{B}$, and that completes the proof of the theorem.

(2.9) Corollary. There exists a free Boolean equality on $\Delta$ i.e., there exists a unique Boolean equality $(B, E)$ on $\Delta$ having the property that any other Boolean equality on $\Delta$ is a quotient of $(B, E)$.

Proof. The dual Boolean equality of the space $\Phi$ of all equivalence relations on $\Delta$ is the required Boolean equality.

3. Boolean equalities and transformations. In this section, as always, $\Delta$ shall be a fixed nonempty set and $\Phi$ shall be the Boolean space of all equivalence relations on $\Delta$. A function from $\Delta$ into $\Delta$ shall be called a transformation (on $\Delta$ ). The purpose of this section is to study the effect of transformations on Boolean equalities. We begin by extending transformations $\eta$ on $\Delta$ to transformations $\bar{\eta}$ on $\Phi$ by

$$
\bar{\eta}(\phi)(s, t)=\phi(\eta s, \eta t)
$$

for all $s, t$ in $\Delta$. The mapping $\eta \rightarrow \bar{\eta}$ sends the identity onto the identity and $\eta \xi \rightarrow \bar{\xi} \bar{\eta}$ for all transformations $\eta$ and $\xi$ on $\Delta$. Moreover, 


$$
\bar{\eta}^{-1}\{\phi: \phi \in \Phi, \phi(s, t)=1\}=\{\phi: \phi \in \Phi, \phi(\eta s, \eta t)=1\}
$$

for all $s, t$ in $\Delta$. This implies that $\bar{\eta}$ is continuous on $\Phi$ for all transformations $\eta$ on $\Delta$. Suppose $\Psi$ is a subset of $\Phi$ and $G$ is a set of transformations on $\Delta$. We shall say that $\Psi$ is invariant under $G$ or that $G$ leaves $\Psi$ invariant if $\bar{\eta}(\Psi) \subseteq \Psi$ for all $\eta$ in $G$. This last concept allows us to formulate the main theorem of this section.

(3.3) Theorem. Suppose $(B, E)$ is a Boolean equality on $\Delta$ and suppose $\eta$ is a transformation on $\Delta$. Then there exists a (necessarily unique) endomorphism $S(\eta)$ of $B$ so that

$$
S(\eta) E(s, l)=E(\eta s, \eta t)
$$

for all $s, t$ in $\Delta$, if and only if the dual equivalence relation space of $(B, E)$ is invariant under $\eta$.

Proof. We may as well assume that $(B, E)$ is the dual Boolean equality of some Boolean subspace $\Psi$ of $\Phi$. Assume first that $\Psi$ is invariant under $\eta$ i.e., $\bar{\eta}(\Psi) \subseteq \Psi$. Since $\bar{\eta}$ is continuous, it follows from the Stone duality theory that $\bar{\eta}$ induces an endomorphism $S(\eta)$ of $B$. By definition, $S(\eta) p=\bar{\eta}^{-1} p$ for every $p$ in $B$ (remember that the elements of $B$ are the clopen subsets of $\Psi$ ). Therefore, for all $s$ and $t$ in $\Delta, S(\eta) E(s, t)=E(\eta s, \eta t)$ which completes one part of the theorem. Conversely, assume there exists a (necessarily unique) endomorphism $S(\eta)$ of $B$ so that $S(\eta) E(s, t)=E(\eta s, \eta t)$ for all $s$ and $t$ in $\Delta$. Let $\chi$ be the mapping defined by (2.5). If $\phi \in \Phi$ and $v$ is a valuation of $B$ so that $\chi(v)=\phi$, then $\chi(v S(\eta))=\bar{\eta} \phi$ which shows that $\bar{\eta} \phi \in \Psi$ and therefore $\Psi$ is invariant under $\eta$.

(3.5) Corollary. Suppose $(B, E)$ is a Boolean equality on $\Delta, \Psi$ the dual equivalence relation space of $(B, E)$ and $G$ the semi-group of transformations on $\Delta$ which leave $\Psi$ invariant. Then there exists a unique mapping $S$ from $G$ into endomorphisms $S(\eta)$ of $B$ so that $S(\eta) E(s, t)$ $=E(\eta s, \eta t)$ for all $\eta$ in $G$ and all $s, t$ in $\Delta$. Moreover, the mapping $S$ has the following two properties: (3.6) If $\delta$ is the identity on $\Delta$, then $S(\delta)$ is the identity on $B ;(3.7)$ If $\eta$ and $\xi$ are in $G$, then $S(\eta \xi)=S(\eta) S(\xi)$.

4. Boolean equalities and operations. If $\Delta$ is a nonempty set and $n$ is a positive integer, then a mapping from $\Delta^{n}$ into $\Delta$ shall be called an $n$-operation or simply an operation on $\Delta$. The purpose of this section is to establish the connection between operations on $\Delta$ and Boolean equalities on $\Delta$.

Suppose $(B, E)$ is a Boolean equality on $\Delta$ and $T$ is an $n$-operation. Then $(B, E)$ is said to be compatible with $T$ if 


$$
E\left(s_{1}, t_{1}\right) \wedge \cdots \wedge E\left(s_{n}, t_{n}\right) \leqq E\left(T\left(s_{1}, \cdots, s_{n}\right), T\left(t_{1}, \cdots, t_{n}\right)\right)
$$

for all $s_{1}, \cdots, s_{n}, t_{1}, \cdots, t_{n}$ in $\Delta ; E$ itself is said to be compatible with $T$ if $(B, E)$ is compatible with $T$.

It is easy to see that if $T$ is an operation on $\Delta$, then the space of equivalence relations compatible with $T$ is compact. This, combined with the following theorem, implies that there exists a free Boolean equality on $\Delta$ which is compatible with $T$ (i.e., free with respect to the property of being compatible with $T$ ).

(4.1) Theorem. Suppose $(B, E)$ is a Boolean equality on a set $\Delta$ and suppose $\Psi$ is the dual equivalence relation space of $(B, E)$. If $T$ is an operation on $\Delta$, then $(B, E)$ is compatible with $T$ if and only if every equivalence relation $\phi$ in $\Psi$ is compatible with $T$.

Proof. Using (2.2) and (2.3), the proof reduces to a straightforward verification.

We conclude by making a few remarks concerning Boolean equalities on familiar algebraic structures. Suppose, for instance, $(B, E)$ is a Boolean equality on a ring $\Delta$ and assume moreover that $(B, E)$ is compatible with the ring operations. By (4.1), if $\phi$ is in the dual of $(B, E)$, then $\phi$ corresponds to a uniquely determined ideal in $\Delta$. In other words, to every Boolean equality on $\Delta$ compatible with the ring operations there corresponds a uniquely determined set of ideals in $\Delta$. Such sets of ideals are easily characterized.

\section{REFERENCES}

1. P. R. Halmos, The basic concepts of algebraic logic, Amer. Math. Monthly vol. 63 (1956) pp. 363-387.

2. J. Los, Quelques remarques, theorèmes et problemes sur les classes definissables: d'algebres, Mathematical interpretation of formal systems, Amsterdam, North. Holland Publishing Co., 1955, pp. 98-113.

UnIVERSITE DE Montreal 